\title{
BAYES ESTIMATION IN THE HIERARCHICAL MULTINOMIAL PROBIT MODEL
}

\author{
Harunori Mori*
}

\begin{abstract}
We consider a complete hierarchical multinomial probit (HMNP) model in which both the regression-coefficient vector and the covariance matrix are assumed to have hierarchical structure and propose an MCMC algorithm for numerically computing the Bayes estimates of the parameters. We show by simulation studies that the covariance matrix is estimated with higher accuracy using the method proposed in this paper than that using an HMNP model in which the covariance matrix is not assumed to have hierarchical structure.
\end{abstract}

Key words and phrases: Bayes estimation, data augmentation, hierarchical multinomial probit model, Markov chain Monte Carlo.

\section{Introduction}

The multinomial logit (MNL) model is a type of discrete choice model that affords straightforward analysis and is widely used across a variety of disciplines. However, as is well known, the MNL model suffers from the prominent drawback known as the assumption of independence of irrelevant alternatives. One discrete choice model that avoids this problem is the multinomial probit (MNP) model. Markov chain Monte Carlo (MCMC) algorithms for numerically computing the Bayes estimates of the parameters in the MNP model have been developed by McCulloch and Rossi (1994), Nobile (1998), McCulloch et al. (2000), and Imai and van Dyk (2005).

When Bayes methods are used to estimate simultaneously the regressioncoefficient vector and the covariance matrix for the results of discrete choices made by multiple individuals with separate MNP models hypothesized for each individual, the hyperparameters of the prior distribution become estimands as well. In this case, because the prior distributions of the hyperparameters are required, the prior distributions of the parameters are represented in a hierarchical fashion, resulting in the hierarchical multinomial probit (HMNP) model. The reason for estimating the regression-coefficient vector and the covariance matrix simultaneously is that such a procedure offers the possibility of smaller estimation errors than simply estimating on the basis of the MNP model for each individual separately.

Nonetheless, to date there have been very few studies treating the HMNP model. The original study by McCulloch and Rossi (1994), which pioneered the concept of Bayes estimation in MNP models, discussed the HMNP model as an

\footnotetext{
Received April 7, 2014. Revised September 29, 2014. Accepted October 15, 2014.

*Faculty of Urban Liberal Arts, Tokyo Metropolitan University, Minami-osawa 1-1, Hachioji-shi, Tokyo 192-0397, Japan.
} 
application of this concept. However, these authors assumed a hierarchical structure only for the regression-coefficient vector, with the covariance matrix shared in common by all individuals. In both the study by Allenby and Rossi (1999), which considered the HMNP model from the perspective of understanding consumer heterogeneity, and the study by Rossi et al. (1996), which considered a multivariate linear regression of the regression-coefficient vector on demographic variables, the covariance matrix not only was shared in common by all individuals, but was further assumed to be a diagonal matrix.

Thus, there has yet to be reported a study considering a complete HMNP model in which both the regression-coefficient vector and the covariance matrix are assumed to have hierarchical structure. In the present study, we consider a complete HMNP model and propose an MCMC algorithm for numerically computing the Bayes estimates of the parameters. We show by simulation studies that the covariance matrix is estimated with higher accuracy using the method proposed in this paper than that using an HMNP model in which the covariance matrix is not assumed to have hierarchical structure.

The remainder of this paper is organized as follows. In the following section, we outline our proposed HMNP model, and in Section 3, we discuss the corresponding sampling algorithms. In Section 4, we present simulation results, and in Section 5, we present empirical applications using sales data.

\section{Bayes estimation in the HMNP model}

In this paper, we will use the following notation for probability distributions. The p-variate normal distribution with mean vector $\boldsymbol{\mu}$ and covariance matrix $\boldsymbol{\Sigma}$ is denoted by $N_{p}(\boldsymbol{\mu}, \boldsymbol{\Sigma})$. The $p$ th-order Wishart distribution with degrees of freedom $\nu$ and covariance matrix $\boldsymbol{\Psi}$ is $W_{p}(\nu, \boldsymbol{\Psi})$, and the corresponding inverted Wishart distribution is $W_{p}^{-1}\left(\nu, \Psi^{-1}\right)$. The gamma distribution with shape parameter $\nu$ and scale parameter $\psi$ is denoted by $G a(\nu, \psi)$.

The density function of a continuous random variable $X$ is denoted as $\pi(x)$, and similarly, the joint and conditional density functions are represented by $\pi(x, y)$ and $\pi(x \mid y)$, respectively. When we must refer to multiple variables $x_{i}, i=1,2, \ldots, n$, for example the notation for posterior distributions, these will be abbreviated as $\left\{x_{i}\right\}$.

First, we define the simplest HMNP model in which the covariance matrix was shared in common by all individuals. We will refer to this HMNP model as Model 1. Second, we define a HMNP model in which the covariance matrix is defined separately for each individual, but is not assumed to exhibit a hierarchical structure. We will refer to this HMNP model as Model 2. Finally we define the complete HMNP model in which both the regression-coefficient vector and the covariance matrix are assumed to have hierarchical structure. We will refer to this HMNP model proposed in this paper as Model 3.

\subsection{Definition of Model 1}

The result of the $t$ th choice made by individual $c$ from among $p$ choice alternatives, which is denoted as $Y_{c t}$, may be defined as follows in terms of latent 
variables $\tilde{W}_{c t i}, i=1,2, \ldots, p-1$ :

$$
Y_{c t}= \begin{cases}p ; & \max \left\{\tilde{W}_{c t 1}, \ldots, \tilde{W}_{c t, p-1}\right\}<0 \\ i ; & \max \left\{\tilde{W}_{c t 1}, \ldots, \tilde{W}_{c t, p-1}\right\}=\tilde{W}_{c t i}>0\end{cases}
$$

for $t=1,2, \ldots, T_{c}, c=1,2, \ldots, C$. The column vector with elements $\tilde{W}_{c t i}$, $i=1,2, \ldots, p-1$ is denoted by $\tilde{\boldsymbol{W}}_{c t}$, which is defined by

$$
\tilde{\boldsymbol{W}}_{c t} \mid \tilde{\boldsymbol{\beta}}_{c}, \tilde{\boldsymbol{\Sigma}} \sim N_{p-1}\left(\boldsymbol{X}_{c t} \tilde{\boldsymbol{\beta}}_{c}, \tilde{\boldsymbol{\Sigma}}\right), \quad t=1,2, \ldots, T_{c}, c=1,2, \ldots, C,
$$

where $\boldsymbol{X}_{c t}$ is the $(p-1) \times r$ covariate matrix and $\tilde{\boldsymbol{\beta}}_{c}$ is the vector of their regression coefficients.

In this probability model, there is an identification problem referred to as multiplicative redundancy. To solve this problem, following standard practice, Imai and van Dyk (2005) set $u^{2}=\tilde{\sigma}_{11}$ and took

$$
\boldsymbol{\beta}_{c}=u^{-1} \tilde{\boldsymbol{\beta}}_{c}, \quad c=1,2, \ldots, C, \quad \boldsymbol{\Sigma}=u^{-2} \tilde{\boldsymbol{\Sigma}}
$$

to be estimands. The $(1,1)$ element of $\boldsymbol{\Sigma}$ was fixed at 1 . However, even if $E\left[\tilde{\sigma}_{11}\right]=E\left[\tilde{\sigma}_{22}\right]$ in the prior distribution, in general, $E\left[\sigma_{22}\right]=1$ does not hold. To avoid this unnatural behavior, in the present study, the proposal of Burgette and Nordheim (2012) is adopted; rather than fixing the value of a specific element of $\boldsymbol{\Sigma}$, we fix the average of the diagonal elements of $\boldsymbol{\Sigma}$ to be equal to 1 and define

$$
u^{2}=\frac{1}{p-1} \operatorname{tr} \tilde{\boldsymbol{\Sigma}}
$$

Upon defining $\boldsymbol{W}_{c t}=u^{-1} \tilde{\boldsymbol{W}}_{c t}$ for each $c$ and $t$, the model (2.2) may be expressed as follows:

$$
\boldsymbol{W}_{c t} \mid \boldsymbol{\beta}_{c}, \boldsymbol{\Sigma} \sim N_{p-1}\left(\boldsymbol{X}_{c t} \boldsymbol{\beta}_{c}, \boldsymbol{\Sigma}\right), \quad t=1,2, \ldots, T_{c}, \quad c=1,2, \ldots, C .
$$

To obtain the equation that defines $Y_{c t}$ corresponding to $\boldsymbol{W}_{c t}$, we simply replace $\tilde{\boldsymbol{W}}_{c t}$ by $\boldsymbol{W}_{c t}$ in equation (2.1).

In this study, we used the prior distribution proposed by Imai and van Dyk (2005) and by Burgette and Nordheim (2012) as the prior distribution of the regression-coefficient vector and the covariance matrix.

The prior distribution of the regression-coefficient vector $\tilde{\boldsymbol{\beta}}_{c}$ in model (2.2) is assumed to be

$$
\tilde{\boldsymbol{\beta}}_{c} \mid \tilde{\boldsymbol{\Sigma}}, \boldsymbol{\mu}, \boldsymbol{T} \sim N_{r}\left(u \boldsymbol{\mu}, u^{2} \boldsymbol{T}\right), \quad c=1,2, \ldots, C .
$$

Then the prior distribution of $\boldsymbol{\beta}_{c}$ is $\boldsymbol{\beta}_{c} \mid \boldsymbol{\mu}, \boldsymbol{T} \sim N_{r}(\boldsymbol{\mu}, \boldsymbol{T})$. The prior distributions of the hyperparameters are assumed to be

$$
\boldsymbol{\mu} \sim N_{r}\left(\mathbf{0}, \mathbf{\Psi}_{\mu}\right), \quad \boldsymbol{T} \sim W_{r}^{-1}\left(\nu_{T}, \mathbf{\Psi}_{T}\right) .
$$


The prior distribution of the covariance matrix $\tilde{\boldsymbol{\Sigma}}$ is assumed to be

$$
\tilde{\boldsymbol{\Sigma}} \mid \delta, \boldsymbol{\Phi} \sim W_{p-1}^{-1}(\delta,(\delta-p) \boldsymbol{\Phi}),
$$

where the degrees of freedom $\delta$ satisfies the condition $\delta>p$. The mean and variance of $\tilde{\boldsymbol{\Sigma}}$ are as follows:

$$
\begin{aligned}
E[\tilde{\boldsymbol{\Sigma}} \mid \delta, \boldsymbol{\Phi}] & =\boldsymbol{\Phi} \\
V\left[\tilde{\sigma}_{i i} \mid \delta, \boldsymbol{\Phi}\right] & =\frac{2 \phi_{i i}^{2}}{\delta-p-2}, \\
V\left[\tilde{\sigma}_{i j} \mid \delta, \boldsymbol{\Phi}\right] & =\frac{(\delta-p+2) \phi_{i j}^{2}+(\delta-p) \phi_{i i} \phi_{j j}}{(\delta-p+1)(\delta-p-2)}, \quad i \neq j .
\end{aligned}
$$

This derivation follows Haff (1979). The prior distribution of $\boldsymbol{\Sigma}$ is not a standard distribution. The density function is given as follows:

$$
\begin{aligned}
\pi(\boldsymbol{\Sigma} \mid \delta, \boldsymbol{\Phi})= & \Gamma\left(\frac{(p-1) \delta}{2}\right) \\
& \times \Gamma_{p-1}^{-1}\left(\frac{\delta}{2}\right)|\boldsymbol{\Phi}|^{\delta / 2}|\boldsymbol{\Sigma}|^{-(\delta+p) / 2}\left(\operatorname{tr} \boldsymbol{\Phi} \boldsymbol{\Sigma}^{-1}\right)^{-(p-1) \delta / 2}
\end{aligned}
$$

where $\Gamma_{p-1}\left(\frac{\delta}{2}\right)$ denotes the multivariate Gamma distribution (Anderson (2003)).

As explained in the following section, what is required for a Gibbs sampler is not the prior distribution (2.10) but rather the prior distribution of the parameter $u^{2}$. This is

$$
u^{2} \mid \boldsymbol{\Sigma}, \delta, \boldsymbol{\Phi} \sim W_{1}^{-1}\left((p-1) \delta,(\delta-p) \operatorname{tr} \mathbf{\Phi} \boldsymbol{\Sigma}^{-1}\right) .
$$

\subsection{Definition of Model 2}

In Model 2, since the covariance matrix is defined separately for each individual, the defining equation (2.2) for $\tilde{\boldsymbol{W}}_{c t}$ takes the form

$$
\tilde{\boldsymbol{W}}_{c t} \mid \tilde{\boldsymbol{\beta}}_{c}, \tilde{\boldsymbol{\Sigma}}_{c} \sim N_{p-1}\left(\boldsymbol{X}_{c t} \tilde{\boldsymbol{\beta}}_{c}, \tilde{\boldsymbol{\Sigma}}_{c}\right), \quad t=1,2, \ldots, T_{c}, c=1,2, \ldots, C .
$$

Upon defining

$$
\boldsymbol{W}_{c t}=u_{c}^{-1} \tilde{\boldsymbol{W}}_{c t}, \quad u_{c}^{2}=\frac{1}{p-1} \operatorname{tr} \tilde{\boldsymbol{\Sigma}}_{c}, \quad t=1,2, \ldots, T_{c}, \quad c=1,2, \ldots, C,
$$

the model (2.11) may be expressed as follows:

$$
\boldsymbol{W}_{c t} \mid \boldsymbol{\beta}_{c}, \boldsymbol{\Sigma}_{c} \sim N_{p-1}\left(\boldsymbol{X}_{c t} \boldsymbol{\beta}_{c}, \boldsymbol{\Sigma}_{c}\right), \quad t=1,2, \ldots, T_{c}, c=1,2, \ldots, C,
$$

where

$$
\boldsymbol{\beta}_{c}=u_{c}^{-1} \tilde{\boldsymbol{\beta}}_{c}, \quad \boldsymbol{\Sigma}_{c}=u_{c}^{-2} \tilde{\boldsymbol{\Sigma}}_{c}, \quad c=1,2, \ldots, C,
$$

which are estimands. 
The prior distribution of $\tilde{\boldsymbol{\beta}}_{c}$ in model (2.11) is assumed to be

$$
\tilde{\boldsymbol{\beta}}_{c} \mid \tilde{\boldsymbol{\Sigma}}_{c}, \boldsymbol{\mu}, \boldsymbol{T} \sim N_{r}\left(u_{c} \boldsymbol{\mu}, u_{c}^{2} \boldsymbol{T}\right), \quad c=1,2, \ldots, C .
$$

Then we have $\boldsymbol{\beta}_{c} \mid \boldsymbol{\mu}, \boldsymbol{T} \sim N_{r}(\boldsymbol{\mu}, \boldsymbol{T})$. For $\boldsymbol{\mu}$ and $\boldsymbol{T}$, we assume the prior distributions (2.5), which are the same as in Model 1. The prior distribution of the covariance matrix $\tilde{\boldsymbol{\Sigma}}_{c}$ is assumed to be

$$
\tilde{\boldsymbol{\Sigma}}_{c} \mid \delta, \boldsymbol{\Phi} \sim W_{p-1}^{-1}(\delta,(\delta-p) \mathbf{\Phi}), \quad c=1,2, \ldots, C .
$$

\subsection{Definition of Model 3}

In Model 3, since the covariance matrix is assumed to have hierarchical structure, we must specify the prior distribution of $\delta$ and $\boldsymbol{\Phi}$, which are the hyperparameters of the prior distribution (2.14). We constructed prior distributions of $\delta$ and $\boldsymbol{\Phi}$ following Asano et al. (2013).

The degrees of freedom $\delta$ satisfies the condition $\delta>d>p$, and we assumed a Gamma distribution for the quantity $\delta-d$ :

$$
\delta-d \sim G a\left(\nu_{\delta}, \psi_{\delta}\right) .
$$

It follows immediately from (2.10) that $\pi\left(\boldsymbol{\Sigma}_{c} \mid \delta, \boldsymbol{\Phi}\right)=\pi\left(\boldsymbol{\Sigma}_{c} \mid \delta, \hat{\mathbf{\Phi}}\right)$, where $\hat{\boldsymbol{\Phi}}=a \boldsymbol{\Phi}, a>0$. This means that $\boldsymbol{\Phi}$ is not identified given $\left\{\boldsymbol{\Sigma}_{c}\right\}$. For this reason, in the present study, we impose the identification condition $\operatorname{tr} \boldsymbol{\Phi}=p-1$, similarly to what is done for $\boldsymbol{\Sigma}_{c}$.

The prior distribution of the precision matrix $\boldsymbol{\Phi}$ is constructed as follows, using ideas similar to those used for $\boldsymbol{\Sigma}_{c}$. First, we set

$$
\hat{\boldsymbol{\Phi}} \sim W_{p-1}\left(\nu_{\Phi}, \Psi_{\Phi}\right),
$$

and define $\mathbf{\Phi}=v^{-2} \hat{\boldsymbol{\Phi}}, v^{2}=\frac{1}{p-1} \operatorname{tr} \hat{\mathbf{\Phi}}$, where

$$
v^{2} \mid \boldsymbol{\Phi} \sim W_{1}\left((p-1) \nu_{\Phi},\left(\operatorname{tr} \boldsymbol{\Psi}_{\Phi}^{-1} \mathbf{\Phi}\right)^{-1}\right),
$$

and the density function for $\boldsymbol{\Phi}$ is

$$
\pi(\boldsymbol{\Phi})=\Gamma\left(\frac{(p-1) \nu_{\Phi}}{2}\right) \Gamma_{p-1}^{-1}\left(\frac{\nu_{\Phi}}{2}\right)\left|\boldsymbol{\Psi}_{\Phi}\right|^{-\nu_{\Phi} / 2}|\boldsymbol{\Phi}|^{\left(\nu_{\Phi}-p\right) / 2}\left(\operatorname{tr} \boldsymbol{\Psi}_{\Phi}^{-1} \mathbf{\Phi}\right)^{-(p-1) \nu_{\Phi} / 2} .
$$

However, this density function is not needed for the Gibbs sampler.

\subsection{Other approaches}

As noted above, the present study builds on the work of Imai and van Dyk (2005), Burgette and Nordheim (2012), and Asano et al. (2013). Of course, other studies have also considered Bayes estimation in the MNP model, and so we here briefly discuss our reasons for choosing not to use previously proposed prior distributions.

McCulloch and Rossi (1994) proposed an HMNP model in which the covariance matrix is common to all individuals. In terms of the notation herein, 
this model has $\tilde{\boldsymbol{W}}_{c t} \mid \tilde{\boldsymbol{\beta}}_{c}, \tilde{\boldsymbol{\Sigma}} \sim N_{p-1}\left(\boldsymbol{X}_{c t} \tilde{\boldsymbol{\beta}}_{c}, \tilde{\boldsymbol{\Sigma}}\right)$, and the prior distribution of $\tilde{\boldsymbol{\beta}}_{c}$ is $N_{r}(\boldsymbol{\mu}, \boldsymbol{T})$. The hyperparameters that may be identified are $u^{-1} \boldsymbol{\mu}$ and $u^{-2} \boldsymbol{T}$, where $u^{2}=\tilde{\sigma}_{11}$. However, if the covariance matrix in these authors' HMNP model is defined on an individual-by-individual basis, then $u_{c}^{-1} \boldsymbol{\mu}$ and $u_{c}^{-2} \boldsymbol{T}$ become estimands, and it is no longer possible to estimate values that are common between individuals: only ratios of elements may be identified. This means that the probability distribution of the identifiable parameters $\boldsymbol{\beta}_{c}$ cannot be known, and thus the model must be considered an incomplete HMNP model.

In an effort to eliminate the multiplicative redundancy in the MNP model, McCulloch et al. (2000) represented the covariance matrix in the form

$$
\tilde{\boldsymbol{\Sigma}}=\left(\begin{array}{cc}
1 & \gamma^{\prime} \\
\gamma & \boldsymbol{\Phi}+\gamma \gamma^{\prime}
\end{array}\right),
$$

where the prior distribution of $\boldsymbol{\gamma}$ is a normal distribution and an inverted Wishart distribution is assumed for $\boldsymbol{\Phi}$. It is possible to extend the MNP model considered by McCulloch et al. to the HMNP model. However, as the authors themselves acknowledge, the Gibbs sampler that they propose exhibits slow decay of the autocorrelation coefficients in the Markov chain, thus ensuring poor efficiency. The extension to the HMNP model requires not only the prior distribution of $\gamma$ but also the prior distribution of its hyperparameters; this makes sampling in this model more cumbersome than in the HMNP model proposed in the present paper.

Working from the perspective of shrinkage estimation for the covariance matrix, Bouriga and Féron (2013) proposed a prior distribution different from that of Asano et al. (2013) for the hyperparameter in an inverted Wishart distribution. These authors showed by simulation studies that, in simultaneous estimation of the covariance matrice, the Bayes estimates obtained using their proposed prior distribution exhibit higher estimation accuracy than those of the maximum likelihood estimators.

If the prior distribution proposed by these authors (their Model 1) were applied to the HMNP model, the prior distribution of $\tilde{\boldsymbol{\Sigma}}_{c}$ would take the form $W_{p-1}^{-1}(\delta, \boldsymbol{\Phi}), \boldsymbol{\Phi}=(\delta-p) \phi \boldsymbol{I}_{p-1}$, where $\phi>0, \delta>p$, and $\boldsymbol{I}_{p-1}$ is the $(p-1)$ dimensional identity matrix. The prior distribution of the hyperparameters is $\pi(\delta) \propto \delta^{-a}, \pi(\phi) \propto \phi^{-1}$, where $a$ is a natural number. However, we cannot apply their prior distribution here for the following reasons. First, the prior distribution $\pi(\phi)$ is improper; furthermore, $\pi(\delta)$ is also improper for $a=1$ and the variance does not exist unless $a \geq 4$. The matrix $\tilde{\boldsymbol{\Sigma}}_{c}$ sampled under such a non-informative prior distribution tends to become singular, whereupon the Gibbs sampler does not work well. On the other hand, choosing large values of $a$ ensures that the average value of $\delta$ approaches $p$ and that its variance tends rapidly to 0 , resulting in an extremely unnatural prior distribution.

Originally, the MNP model was defined as follows:

$$
\begin{aligned}
& Y_{t}=i \Longleftrightarrow \max \left\{\tilde{W}_{t 1}^{*}, \ldots, \tilde{W}_{t p}^{*}\right\}=\tilde{W}_{t i}^{*}, \quad i=1,2, \ldots, p, \\
& \tilde{\boldsymbol{W}}_{t}^{*} \mid \tilde{\boldsymbol{\beta}}^{*}, \tilde{\boldsymbol{\Sigma}}^{*} \sim N_{p}\left(\boldsymbol{X}_{t}^{*} \tilde{\boldsymbol{\beta}}^{*}, \tilde{\boldsymbol{\Sigma}}^{*}\right) .
\end{aligned}
$$


This probability model exhibits additive redundancy, and hence does not allow parameter identification. To remedy this problem, we perform the replacements $\tilde{W}_{t i}=\tilde{W}_{t i}^{*}-\tilde{W}_{t p}^{*}, i=1,2, \ldots, p-1$, which results in equations (2.1) and (2.2). The covariance matrix then satisfies the relation $\tilde{\boldsymbol{\Sigma}}=\boldsymbol{D} \tilde{\boldsymbol{\Sigma}}^{*} \boldsymbol{D}^{\prime}$, where $\boldsymbol{D}=$ $\left(\boldsymbol{I}_{p-1}-\mathbf{1}_{p-1}\right)$; here $\mathbf{1}_{p-1}$ is the $(p-1) \times 1$ vector with all elements equal to 1 . If we assume that $\tilde{\boldsymbol{\Sigma}}^{*} \sim W_{p}^{-1}\left(\delta^{*}, \boldsymbol{\Phi}^{*}\right)$, then $\tilde{\boldsymbol{\Sigma}} \sim W_{p-1}^{-1}\left(\delta^{*}-1, \boldsymbol{D} \boldsymbol{\Phi}^{*} \boldsymbol{D}^{\prime}\right)$. This is the prior distribution (2.6), and so $\delta=\delta^{*}-1, \boldsymbol{\Phi}=\boldsymbol{D} \boldsymbol{\Phi}^{*} \boldsymbol{D}^{\prime}$. Consequently, we find $\boldsymbol{\Phi} \propto \boldsymbol{I}_{p-1}$, resulting again in an extremely unnatural hypothesis.

In contrast to Bouriga and Féron (2013), in the present study, the traceadjusted precision matrix $\boldsymbol{\Phi}$ is also an important estimand. However, with the above prior distribution, we would have simply $\boldsymbol{\Phi}=\boldsymbol{I}_{p-1}$, whereupon $\boldsymbol{\Phi}$ ceases to be an estimand at all.

\section{Sampling algorithms}

\subsection{Sampling algorithm of Model 1}

In the present study, except for sampling $\delta$, we may use the Gibbs sampler as an MCMC method. However, because we do not assume a conjugate prior distribution for $\delta$, we must sample its value using a Metropolis-Hastings step.

In Model 1, we may use the Gibbs sampler as an MCMC method. The sampling algorithm proceeds as follows.

(i) Draw $\boldsymbol{W}_{c t} \mid \boldsymbol{Y}_{c t}, \boldsymbol{\beta}_{c}, \boldsymbol{\Sigma}$ independently for $t=1,2, \ldots, T_{c}, c=1,2, \ldots, C$.

This step is composed of $p-1$ conditional draws from a truncated normal distribution, as discussed in McCulloch and Rossi (1994).

(ii) Draw $\{\boldsymbol{\Sigma}\}$.

(a) For each $c$, we draw $u^{2}\left|*=u^{2}\right| \boldsymbol{\Sigma}$. The notation $u^{2} \mid *$ indicates that all parameters other than $u^{2}$ and all latent variables are conditions. We will use this notation below to convey a similar meaning.

(b) Set $\tilde{\boldsymbol{e}}_{c t}=u\left(\boldsymbol{W}_{c t}-\boldsymbol{X}_{c t} \boldsymbol{\beta}_{c}\right)$; then $\tilde{\boldsymbol{e}}_{c t} \mid * \sim N_{p-1}(\mathbf{0}, \tilde{\boldsymbol{\Sigma}})$. Thus, we use $\left\{\tilde{\boldsymbol{e}}_{c t}\right\}$ as conditions instead of $\left\{\boldsymbol{W}_{c t}\right\}$, and we draw $\tilde{\boldsymbol{\Sigma}}$. The posterior distribution takes the following form:

$$
\tilde{\boldsymbol{\Sigma}}|*=\tilde{\boldsymbol{\Sigma}}|\left\{\tilde{\boldsymbol{e}}_{c t}\right\} \sim W_{p-1}^{-1}(\nu, \mathbf{\Psi}),
$$

where $\nu=\delta+\sum_{c=1}^{C} T_{c}, \boldsymbol{\Psi}=(\delta-p) \boldsymbol{\Phi}+\sum_{c=1}^{C} \sum_{t=1}^{T_{c}} \tilde{\boldsymbol{e}}_{c t} \tilde{\boldsymbol{e}}_{c t}^{\prime}$.

(c) We set $u^{2}=\frac{1}{p-1} \operatorname{tr} \tilde{\boldsymbol{\Sigma}}$ and $\boldsymbol{\Sigma}=u^{-2} \tilde{\boldsymbol{\Sigma}}$. In addition, for each $t$, we set $\boldsymbol{W}_{c t}=u^{-1} \tilde{\boldsymbol{e}}_{c t}+\boldsymbol{X}_{c t} \boldsymbol{\beta}_{c}$.

(iii) Draw $\boldsymbol{\beta}_{c} \mid * \sim N_{r}\left(\boldsymbol{A}_{c} \boldsymbol{b}_{c}, \boldsymbol{A}_{c}\right)$ independently for $c=1,2, \ldots, C$.

In this equation, we have $\boldsymbol{A}_{c}^{-1}=\sum_{t=1}^{T_{c}} \boldsymbol{X}_{c t}^{\prime} \boldsymbol{\Sigma}^{-1} \boldsymbol{X}_{c t}+\boldsymbol{T}^{-1}, \quad \boldsymbol{b}_{c}=$ $\sum_{t=1}^{T_{c}} \boldsymbol{X}_{c t}^{\prime} \boldsymbol{\Sigma}^{-1} \boldsymbol{W}_{c t}+\boldsymbol{T}^{-1} \boldsymbol{\mu}$.

(iv) Draw $\boldsymbol{\mu} \mid * \sim N_{r}(\boldsymbol{A} \boldsymbol{b}, \boldsymbol{A})$.

Here $\boldsymbol{A}^{-1}=C \boldsymbol{T}^{-1}+\boldsymbol{\Psi}_{\mu}^{-1}, b=\boldsymbol{T}^{-1} \sum_{c=1}^{C} \boldsymbol{\beta}_{c}$.

(v) Draw $\boldsymbol{T} \mid * \sim W_{r}^{-1}(\nu, \boldsymbol{\Psi})$.

Here $\nu=\nu_{T}+C, \boldsymbol{\Psi}=\boldsymbol{\Psi}_{T}+\sum_{c=1}^{C}\left(\boldsymbol{\beta}_{c}-\boldsymbol{\mu}\right)\left(\boldsymbol{\beta}_{c}-\boldsymbol{\mu}\right)^{\prime}$.

Steps 1, 2 and 3 apply Algorithm 2 of Imai and van Dyk (2005) to each individual. These authors proposed their Algorithm 2 as a sampling algorithm 
applicable to the case in which $E\left[\boldsymbol{\beta}_{c} \mid \boldsymbol{\mu}, \boldsymbol{T}\right] \neq \mathbf{0}$.

\subsection{Sampling algorithm of Model 2}

To define a sampling algorithm for Model 2, it suffices to modify Step 2 as follows.

(ii) Draw $\left\{\boldsymbol{\Sigma}_{c}\right\}$.

(a) For each $c$, draw $u_{c}^{2}\left|*=u_{c}^{2}\right| \boldsymbol{\Sigma}_{c}, \delta, \boldsymbol{\Phi}$.

(b) Set $\tilde{\boldsymbol{e}}_{c t}=u_{c}\left(\boldsymbol{W}_{c t}-\boldsymbol{X}_{c t} \boldsymbol{\beta}_{c}\right)$; then $\tilde{\boldsymbol{e}}_{c t} \mid * \sim N_{p-1}\left(\mathbf{0}, \tilde{\boldsymbol{\Sigma}}_{c}\right)$. Thus, we use $\left\{\tilde{\boldsymbol{e}}_{c t}\right\}$ as conditions instead of $\left\{\boldsymbol{W}_{c t}\right\}$, and we draw $\tilde{\boldsymbol{\Sigma}}_{c}$. The posterior distribution takes the following form:

$$
\tilde{\boldsymbol{\Sigma}}_{c}\left|*=\tilde{\boldsymbol{\Sigma}}_{c}\right|\left\{\tilde{\boldsymbol{e}}_{c t}\right\} \sim W_{p-1}^{-1}\left(\nu_{c}, \mathbf{\Psi}_{c}\right), \quad c=1,2, \ldots, C,
$$

where $\nu_{c}=\delta+T_{c}, \mathbf{\Psi}_{c}=(\delta-p) \boldsymbol{\Phi}+\sum_{t=1}^{T_{c}} \tilde{\boldsymbol{e}}_{c t} \tilde{\boldsymbol{e}}_{c t}^{\prime}$.

(c) For each $c$, we set $u_{c}^{2}=\frac{1}{p-1} \operatorname{tr} \tilde{\boldsymbol{\Sigma}}_{c}$ and $\boldsymbol{\Sigma}_{c}=u_{c}^{-2} \tilde{\boldsymbol{\Sigma}}_{c}$. In addition, for each $t$, we set $\boldsymbol{W}_{c t}=u_{c}^{-1} \tilde{\boldsymbol{e}}_{c t}+\boldsymbol{X}_{c t} \boldsymbol{\beta}_{c}$.

Steps 1 and 2 constitute an algorithm for generating a Markov chain whose stationary distribution has the joint density function $\pi\left(\left\{\boldsymbol{W}_{c t}\right\},\left\{\boldsymbol{\Sigma}_{c}\right\} \mid *\right)$. However, although the authors do not explicitly state this, the sampled values $\boldsymbol{W}_{c t}$ do not always satisfy the conditions restricted by $Y_{c t}$. In the simulations presented in the following section, there were many cases in which the sampling had to be terminated because the matrix $\tilde{\boldsymbol{\Sigma}}_{c}$ sampled in Step 2(b) became singular. Inconsistencies between the sampled values of $\boldsymbol{W}_{c t}$ and $Y_{c t}$ are one possible cause of this phenomenon.

\subsection{Sampling algorithm of Model 2}

To define a sampling algorithm for Model 3, it suffices to add the following steps to the sampling algorithm for Model 2.

(vi) Draw $\boldsymbol{\Phi}$.

(a) For each $c$, we draw $u_{c}^{2}\left|*=u_{c}^{2}\right| \boldsymbol{\Sigma}_{c}, \delta$, $\boldsymbol{\Phi}$ and set $\tilde{\boldsymbol{\Sigma}}_{c}=u_{c}^{2} \boldsymbol{\Sigma}_{c}$.

(b) We draw $v^{2}\left|*=v^{2}\right| \boldsymbol{\Phi}$ and set $\hat{\boldsymbol{\Sigma}}_{c}=v^{2} \tilde{\boldsymbol{\Sigma}}_{c}$ for each $c$. The posterior distribution takes the following form:

$$
\hat{\boldsymbol{\Sigma}}_{c} \mid \delta, \hat{\boldsymbol{\Phi}} \sim W_{p-1}^{-1}(\delta,(\delta-p) \hat{\boldsymbol{\Phi}}),
$$

where $\hat{\mathbf{\Phi}}=v^{2} \mathbf{\Phi}$.

(c) Draw $\hat{\boldsymbol{\Phi}}|*=\hat{\mathbf{\Phi}}|\left\{\hat{\boldsymbol{\Sigma}}_{c}\right\}, \delta \sim W_{p-1}(\nu, \mathbf{\Psi})$.

Here $\nu=C \delta+\nu_{\Phi}, \boldsymbol{\Psi}=\boldsymbol{\Psi}_{\Phi}^{-1}+\sum_{c=1}^{C} \hat{\boldsymbol{\Sigma}}_{c}^{-1}$.

(d) Set $v^{2}=\frac{1}{p-1} \operatorname{tr} \hat{\boldsymbol{\Phi}}$ and $\boldsymbol{\Phi}=v^{-2} \hat{\boldsymbol{\Phi}}$.

(vii) Draw $\delta \mid *$.

Note that $\pi(\delta \mid *) \propto \pi\left(\left\{\boldsymbol{\Sigma}_{c}\right\} \mid \delta, \boldsymbol{\Phi}\right) \pi(\delta)$ is not a standard distribution and requires a Metropolis-Hastings step.

Step 6, which samples $\boldsymbol{\Phi}$, is based on the following rationale. From the definition of the conditional density function,

$$
\pi\left(\boldsymbol{\Phi},\left\{u_{c}^{2}\right\} \mid\left\{\boldsymbol{\Sigma}_{c}\right\}\right)=\pi\left(\boldsymbol{\Phi} \mid\left\{u_{c}^{2}\right\},\left\{\boldsymbol{\Sigma}_{c}\right\}\right) \pi\left(\left\{u_{c}^{2}\right\} \mid\left\{\boldsymbol{\Sigma}_{c}\right\}\right) .
$$


When sampling $\boldsymbol{\Phi} \mid\left\{\boldsymbol{\Sigma}_{c}\right\}$, one should first sample $\left\{u_{c}^{2}\right\} \mid\left\{\boldsymbol{\Sigma}_{c}\right\}$ and then sample $\boldsymbol{\Phi}\left|\left\{u_{c}^{2}\right\},\left\{\boldsymbol{\Sigma}_{c}\right\}=\boldsymbol{\Phi}\right|\left\{\tilde{\boldsymbol{\Sigma}}_{c}\right\}$. However, because it is difficult to sample $\boldsymbol{\Phi} \mid\left\{\tilde{\boldsymbol{\Sigma}}_{c}\right\}$ directly, for the present study, we use the Marginal Augmentation Scheme 1 (Meng and van Dyk (1999), Imai and van Dyk (2005)) with an augmented data model to be $\hat{\boldsymbol{\Sigma}}_{c}=v^{2} \tilde{\boldsymbol{\Sigma}}_{c}$.

\section{Simulations}

\subsection{Simulations of Model 1}

Data and prior distributions In the simulation study, the accuracy of the Bayes estimates was tested using 10 artificially constructed samples. In constructing these samples, the hyperparameter values were kept fixed, but different values of the covariates and the regression-coefficient vectors $\left\{\boldsymbol{\beta}_{c}\right\}$ were chosen for each sample, for the following reason. The estimation accuracy of the MNP model, which only observes the results of choice $\left\{Y_{c t}\right\}$, depends strongly on the values of the covariates and parameters. Consequently, tests of the estimation accuracy obtained for specific fixed values of the covariates and parameters cannot be said to offer sufficiently thorough validation. The ideal experiment would be to consider various combinations of the covariates and the parameters and to test the estimation accuracy using many samples for each combination. However, restrictions on computation time unfortunately prevented us from carrying out such tests for this study.

The number of choice alternatives $p$ was set to 3 and the number of covariates, excluding intercepts, was set to 2 . Thus, $r=(p-1)+2=4$. The number of individuals was $C=200$, and the sample size for all individuals was $T_{c}=50$.

The values of $\left\{\boldsymbol{\beta}_{c}\right\}$ were generated from the following normal distributions:

$$
\begin{array}{ll}
\beta_{c 1} \sim N(0.8,0.16), & \beta_{c 2} \sim N(-0.8,0.16), \\
\beta_{c 3} \sim N(0.3,0.25), & \beta_{c 4} \sim N(0.8,0.25),
\end{array}
$$

where $\beta_{c 3}$ and $\beta_{c 4}$ are intercepts. The covariance matrix was set as $\boldsymbol{\Sigma}=$ $\left(\begin{array}{ll}1.0 & 0.3 \\ 0.3 & 1.0\end{array}\right)$

With the parameters configured as above, we generated samples $\left\{Y_{c t}\right\}$ in accordance with the definition of the MNP model. The covariates were generated from a $N(0,2)$ distribution.

On the other hand, for the estimations, we chose the values of the hyperparameters in the prior distributions so that variances of the parameters to be estimated are sufficiently large. The hyperparameters in the prior distribution (2.5) were set as $\boldsymbol{\Psi}_{\mu}=100 \boldsymbol{I}_{4}, \nu_{T}=7$ and $\boldsymbol{\Psi}_{T}=2 \boldsymbol{I}_{4}$. The variance of $\boldsymbol{T}$ does not exist. The hyperparameters in the prior distribution (2.6) were set as $\delta=7$ and $\boldsymbol{\Phi}=\left(\begin{array}{ll}1.0 & 0.5 \\ 0.5 & 1.0\end{array}\right)$. From equations (2.7) and (2.8), the mean of $\tilde{\boldsymbol{\Sigma}}$ is $\boldsymbol{\Phi}$, and the variance of its diagonal elements is 1 . A variance of 1 cannot be said to be a large value; on the other hand, for $\delta=5$, the variance does not even exist, 
and so this can hardly be considered a strongly informative prior distribution. Moreover, for $\delta=5$, the sampled matrix $\tilde{\boldsymbol{\Sigma}}$ is apt to be singular, and the Gibbs sampler does not work well.

Simulation results We fixed the burn-in period of the Gibbs sampler at 10,000 iterations, and then used the sample constructed during the subsequent 30,000 iterations to compute the posterior means. Convergence of the Markov chain was assessed by testing the hypothesis that the population mean of the first 3,000 iterations and that of the last 15,000 iterations are equal (Geweke (1992)). The standard error of the sample mean was estimated by using the Parzen window with bandwidth of 500 (Matsumae et al. (2011)). For the regression-coefficient vector, we do not separately assess convergence, but rather assess convergence by looking at each element of $\sum_{c=1}^{200} \boldsymbol{\beta}_{c}$. If any parameters for which the hypothesis is rejected are found, then we discard the first 2,000 iterations, run the Gibbs sampler for an additional 2,000 iterations, and reassess convergence. This procedure is repeated until convergence is verified for all parameters.

We assessed whether the number of iterations is too small by using the inefficiency factor (IF). For the variance $\sigma_{11}$, the IF is defined as follows:

$$
\mathrm{IF}=\frac{V\left[\bar{\sigma}_{11} \mid *\right]}{N^{-1} V\left[\sigma_{11} \mid *\right]}, \quad \bar{\sigma}_{11}=\frac{1}{N} \sum_{n=1}^{N} \sigma_{11(n)},
$$

where $N=30,000$, which is the number of iterations, and $\sigma_{11(n)}, n=1,2, \ldots, N$ are the sample generated by the Gibbs sampler. In our simulation, we estimated $V\left[\bar{\sigma}_{11} \mid *\right]$ by using the Parzen window with bandwidth of 500 and $V\left[\sigma_{11} \mid *\right]$ by the sample variance $\frac{1}{N-1} \sum_{n=1}^{N}\left(\sigma_{11(n)}-\bar{\sigma}_{11}\right)^{2}$. Other parameters are treated similarly.

All simulation programs were written in SAS/IML.

Estimation accuracy was assessed for each sample by using the square error (SE). For the variance $\sigma_{11}$, the SE in sample $i$ takes the form $\left(\sigma_{(i) 11}-\hat{\sigma}_{(i) 11}\right)^{2}$, where $\sigma_{(i) 11}$ is the variance $\sigma_{11}$ for sample $i$ and $\hat{\sigma}_{(i) 11}$ is its estimated value. Other parameters except for $\left\{\boldsymbol{\beta}_{c}\right\}$ are treated similarly. Estimation accuracy of $\beta_{c j}, c=1,2, \ldots, 200$ was assessed by using the mean value of the SE computed for each $c$.

Table 1 collects results for the mean value and standard deviation of the SE and the mean value of the IF for each parameter.

Since the sample size per individual is set at the low value of $T_{c}=50$, for $\left\{\boldsymbol{\beta}_{c}\right\}$, the mean values of SE are somewhat large. But, for other parameters, those are sufficiently small. We used the sample of size 30,000 constructed by the Gibbs sampler to compute the posterior means. The values of IF show that this sample corresponds to an independent sample of size not less than 380 .

\subsection{Simulations of Model 3}

Data and prior distributions We consider three settings controlling the construction of covariance matrices. For each setting, we ran the simulation in the 
Table 1. SEs and IFs of parameter estimates.

\begin{tabular}{c|ccc|c|ccr}
\hline \multicolumn{5}{c}{ mean } & s.d. & IF & \multicolumn{3}{c}{ mean } & s.d. & \multicolumn{1}{c}{ IF } \\
\hline$\left\{\beta_{c 1}\right\}$ & 0.03068 & 0.00286 & 76.1 & $\tau_{11}$ & 0.00044 & 0.00054 & 15.9 \\
$\left\{\beta_{c 2}\right\}$ & 0.03094 & 0.00314 & 77.6 & $\tau_{12}$ & 0.00006 & 0.00008 & 11.1 \\
$\left\{\beta_{c 3}\right\}$ & 0.08194 & 0.00870 & 28.2 & $\tau_{13}$ & 0.00003 & 0.00003 & 7.3 \\
$\left\{\beta_{c 4}\right\}$ & 0.08170 & 0.00716 & 26.6 & $\tau_{14}$ & 0.00006 & 0.00006 & 7.4 \\
$\sigma_{11}$ & 0.00270 & 0.00281 & 46.9 & $\tau_{22}$ & 0.00025 & 0.00030 & 16.4 \\
$\sigma_{12}$ & 0.00127 & 0.00321 & 58.4 & $\tau_{23}$ & 0.00014 & 0.00013 & 7.1 \\
$\sigma_{22}$ & 0.00270 & 0.00281 & 46.9 & $\tau_{24}$ & 0.00014 & 0.00021 & 6.8 \\
$\mu_{1}$ & 0.00036 & 0.00033 & 19.0 & $\tau_{33}$ & 0.00028 & 0.00038 & 5.4 \\
$\mu_{2}$ & 0.00152 & 0.00116 & 19.3 & $\tau_{34}$ & 0.00011 & 0.00013 & 4.7 \\
$\mu_{3}$ & 0.00186 & 0.00214 & 12.3 & $\tau_{44}$ & 0.00025 & 0.00028 & 6.3 \\
$\mu_{4}$ & 0.00245 & 0.00218 & 11.0 & & & & \\
\hline \multicolumn{5}{l}{ s.d.: standard deviation. }
\end{tabular}

same way as that of Model 1 in Subsection 4.1. We used different values of not only $\left\{\boldsymbol{\beta}_{c}\right\}$ but $\left\{\boldsymbol{\Sigma}_{c}\right\}$ for each sample.

The covariance matrix $\boldsymbol{\Sigma}_{c}$ was defined by first constructing $\tilde{\boldsymbol{\Sigma}}_{c}$ of the model (2.11) and then adjusting its trace. The covariance matrices $\left\{\tilde{\boldsymbol{\Sigma}}_{c}\right\}$ were generated from the prior distribution (2.14) with the common value $\mathbf{\Phi}=\left(\begin{array}{ll}1.0 & 0.3 \\ 0.3 & 1.0\end{array}\right)$ and three different values of $\delta$, namely, $\delta=15,35$ and 55 . As is clear from equations (2.8) and (2.9), the standard deviation of $\boldsymbol{\Sigma}_{c}$ decreases as $\delta$ increases. Table 4 details the standard deviations in our constructions of $\left\{\sigma_{c 11}\right\}$ and $\left\{\sigma_{c 12}\right\}$.

For the estimations, the hyperparameters in the prior distributions were assigned the same values as in the simulation of Model 1. The hyperparameters in the prior distribution (2.15) were set as $\nu_{\delta}=1.5$ and $\psi_{\delta}=33$. The lower limit of $\delta$, which we denote $d$, was set to 7 in accordance with the simulation of Model 1. However, when the Gibbs sampler did not work well under $d=7$, we used values of $d$ larger than 7 . For $\delta=15$, we set $d=9$ in 3 out of 10 samples and $d=11$ in 2 samples. For $\delta=35$, we set $d=8$ in 1 sample and $d=9$ in 1 sample; for $\delta=55$, we set $d=8$ in 2 samples and $d=9$ in 1 sample. The hyperparameters in the prior distribution $(2.16)$ were set as $\nu_{\Phi}=25, \Psi_{\Phi}=\left(\begin{array}{ll}2 & 1 \\ 1 & 2\end{array}\right)$. The mean of the trace-adjusted precision matrix $\mathbf{\Phi}$ is approximately $\left(\begin{array}{ll}1.000 & 0.485 \\ 0.485 & 1.000\end{array}\right)$, and the variance of its diagonal elements is approximately 0.03 ; both of these values were computed using the Monte Carlo method.

In Step 7 of our sampling algorithm (the Metropolis-Hastings step), the proposal distribution (assuming the present value is $\delta_{(-1)}$ ) was taken to be a uniform distribution with lower bound $\delta_{(-1)}-0.5 \epsilon_{\delta}$ and upper bound $\delta_{(-1)}+0.5 \epsilon_{\delta}$, where $\epsilon_{\delta}$ was determined separately for each sample to ensure an acceptance probability between $30 \%$ and $50 \%$. The mean, standard deviation, minimum, 
Table 2(a). SEs and IFs of parameter estimates $(\delta=15)$.

\begin{tabular}{c|rc|rrr|rrr}
\hline & \multicolumn{2}{|c|}{ Model 1 } & \multicolumn{3}{c|}{ Model 2 } & \multicolumn{3}{c}{ Model 3 } \\
\hline & mean & s.d. & mean & s.d. & \multicolumn{1}{c}{ IF } & mean & s.d. & \multicolumn{1}{c}{ IF } \\
\hline$\left\{\beta_{c 1}\right\}$ & 0.03256 & 0.00227 & 0.05161 & 0.05757 & 100.7 & 0.03351 & 0.00220 & 154.6 \\
$\left\{\beta_{c 2}\right\}$ & 0.03399 & 0.00422 & 0.03914 & 0.01333 & 99.9 & 0.03483 & 0.00414 & 155.2 \\
$\left\{\beta_{c 3}\right\}$ & 0.08781 & 0.00769 & 0.09550 & 0.02238 & 29.9 & 0.08864 & 0.00767 & 40.2 \\
$\left\{\beta_{c 4}\right\}$ & 0.08844 & 0.01250 & 0.08783 & 0.01228 & 31.1 & 0.08971 & 0.01321 & 47.3 \\
\hline$\left\{\sigma_{c 11}\right\}$ & 0.05822 & 0.00432 & 0.05245 & 0.00756 & 18.3 & 0.05009 & 0.00572 & 72.8 \\
$\left\{\sigma_{c 12}\right\}$ & 0.05551 & 0.00581 & 0.06724 & 0.00989 & 19.5 & 0.04918 & 0.00488 & 108.2 \\
$\left\{\sigma_{c 22}\right\}$ & 0.05822 & 0.00432 & 0.05245 & 0.00756 & 18.3 & 0.05009 & 0.00572 & 72.8 \\
\hline$\mu_{1}$ & 0.00050 & 0.00066 & 0.00081 & 0.00110 & 24.4 & 0.00070 & 0.00088 & 46.8 \\
$\mu_{2}$ & 0.00179 & 0.00165 & 0.00077 & 0.00065 & 23.0 & 0.00319 & 0.00360 & 52.3 \\
$\mu_{3}$ & 0.00112 & 0.00157 & 0.00174 & 0.00117 & 12.3 & 0.00106 & 0.00145 & 16.1 \\
$\mu_{4}$ & 0.00225 & 0.00190 & 0.00165 & 0.00173 & 12.2 & 0.00217 & 0.00205 & 19.5 \\
\hline$\tau_{11}$ & 0.00034 & 0.00027 & 0.00487 & 0.01397 & 51.3 & 0.00126 & 0.00113 & 58.5 \\
$\tau_{12}$ & 0.00005 & 0.00005 & 0.00134 & 0.00386 & 46.3 & 0.00019 & 0.00026 & 40.0 \\
$\tau_{13}$ & 0.00013 & 0.00013 & 0.00200 & 0.00566 & 40.5 & 0.00059 & 0.00065 & 29.9 \\
$\tau_{14}$ & 0.00012 & 0.00010 & 0.00012 & 0.00010 & 9.8 & 0.00051 & 0.00053 & 31.3 \\
$\tau_{22}$ & 0.00016 & 0.00019 & 0.00024 & 0.00040 & 45.9 & 0.00061 & 0.00075 & 60.1 \\
$\tau_{23}$ & 0.00013 & 0.00013 & 0.00044 & 0.00099 & 37.0 & 0.00053 & 0.00053 & 29.7 \\
$\tau_{24}$ & 0.00008 & 0.00011 & 0.00008 & 0.00011 & 11.2 & 0.00031 & 0.00055 & 32.4 \\
$\tau_{33}$ & 0.00056 & 0.00064 & 0.00129 & 0.00308 & 28.9 & 0.00255 & 0.00314 & 30.4 \\
$\tau_{34}$ & 0.00019 & 0.00019 & 0.00020 & 0.00019 & 6.3 & 0.00102 & 0.00119 & 24.2 \\
$\tau_{44}$ & 0.00049 & 0.00067 & 0.00033 & 0.00037 & 7.6 & 0.00260 & 0.00397 & 37.2 \\
\hline
\end{tabular}

and maximum of $\epsilon_{\delta}$ were respectively $17.0,10.4,4$, and 36 .

Some care is required in computing the acceptance probability. Denoting the candidate value by $\delta^{\prime}$, if both $\delta_{(-1)}-0.5 \epsilon_{\delta}>d$ and $\delta^{\prime}-0.5 \epsilon_{\delta}>d$ hold, then the sampling process becomes a random walk chain, and the acceptance probability is given by simply $\frac{\pi\left(\delta^{\prime} \mid *\right)}{\pi\left(\delta_{(-1)} \mid *\right)}$. However, since $\delta \geq d$, if $\delta^{\prime}-0.5 \epsilon_{\delta}<d$, then (even if $\delta_{(-1)}-0.5 \epsilon_{\delta}>d$ ) we must compute the acceptance probability as $\frac{\pi\left(\delta^{\prime} \mid *\right)\left(\delta^{\prime}+0.5 \epsilon_{\delta}-d\right)}{\pi\left(\delta_{(-1)} \mid *\right) \epsilon_{\delta}}$.

Simulation results We fixed the burn-in period of the Gibbs sampler at 20,000 iterations, and then used the sample constructed during the subsequent 200,000 iterations to compute the posterior means. Convergence of the Markov chain was assessed by testing the hypothesis that the population mean of the first 20,000 iterations and that of the last 100,000 iterations are equal. The standard error of the sample mean was estimated by using the Parzen window with bandwidth of 2,000 for $\delta$ and 500 for other parameters. We estimated the numerator of the definition of the IF by using the Parzen window with bandwidth of 2,000 for $\delta$ and 500 for other parameters.

For comparison purposes, we also investigated the estimation accuracy of 
Table 2(b). SEs and IFs of parameter estimates $(\delta=35)$.

\begin{tabular}{c|cc|ccc|ccr}
\hline & \multicolumn{2}{|c|}{ Model 1 } & \multicolumn{3}{c|}{ Model 2 } & \multicolumn{3}{c}{ Model 3 } \\
\hline & mean & s.d. & mean & s.d. & \multicolumn{1}{c}{ IF } & mean & s.d. & \multicolumn{1}{c}{ IF } \\
\hline$\left\{\beta_{c 1}\right\}$ & 0.03159 & 0.00353 & 0.03315 & 0.00350 & 92.7 & 0.03234 & 0.00461 & 141.4 \\
$\left\{\beta_{c 2}\right\}$ & 0.03139 & 0.00371 & 0.03230 & 0.00348 & 92.4 & 0.03197 & 0.00402 & 141.0 \\
$\left\{\beta_{c 3}\right\}$ & 0.08433 & 0.01127 & 0.08632 & 0.01189 & 26.9 & 0.08603 & 0.01148 & 39.6 \\
$\left\{\beta_{c 4}\right\}$ & 0.08379 & 0.00786 & 0.08399 & 0.00769 & 32.3 & 0.08527 & 0.00760 & 46.0 \\
\hline$\left\{\sigma_{c 11}\right\}$ & 0.02719 & 0.00393 & 0.03816 & 0.00352 & 20.9 & 0.02680 & 0.00479 & 83.9 \\
$\left\{\sigma_{c 12}\right\}$ & 0.02491 & 0.00355 & 0.04701 & 0.00551 & 21.9 & 0.02452 & 0.00355 & 110.4 \\
$\left\{\sigma_{c 22}\right\}$ & 0.02719 & 0.00393 & 0.03816 & 0.00352 & 20.9 & 0.02680 & 0.00479 & 83.9 \\
\hline$\mu_{1}$ & 0.00110 & 0.00107 & 0.00168 & 0.00187 & 21.5 & 0.00152 & 0.00204 & 42.8 \\
$\mu_{2}$ & 0.00056 & 0.00105 & 0.00143 & 0.00186 & 20.8 & 0.00063 & 0.00111 & 43.7 \\
$\mu_{3}$ & 0.00167 & 0.00167 & 0.00256 & 0.00346 & 11.0 & 0.00180 & 0.00174 & 17.0 \\
$\mu_{4}$ & 0.00146 & 0.00122 & 0.00135 & 0.00134 & 12.7 & 0.00149 & 0.00150 & 18.7 \\
\hline$\tau_{11}$ & 0.00027 & 0.00036 & 0.00015 & 0.00021 & 24.5 & 0.00079 & 0.00108 & 52.9 \\
$\tau_{12}$ & 0.00012 & 0.00014 & 0.00013 & 0.00015 & 16.4 & 0.00031 & 0.00029 & 35.9 \\
$\tau_{13}$ & 0.00006 & 0.00011 & 0.00005 & 0.00010 & 9.5 & 0.00026 & 0.00040 & 26.1 \\
$\tau_{14}$ & 0.00008 & 0.00010 & 0.00007 & 0.00009 & 10.3 & 0.00032 & 0.00045 & 28.5 \\
$\tau_{22}$ & 0.00013 & 0.00013 & 0.00007 & 0.00007 & 22.5 & 0.00035 & 0.00042 & 52.4 \\
$\tau_{23}$ & 0.00009 & 0.00015 & 0.00009 & 0.00016 & 10.7 & 0.00035 & 0.00039 & 26.2 \\
$\tau_{24}$ & 0.00010 & 0.00015 & 0.00010 & 0.00015 & 9.7 & 0.00034 & 0.00049 & 29.1 \\
$\tau_{33}$ & 0.00010 & 0.00012 & 0.00008 & 0.00010 & 6.9 & 0.00052 & 0.00069 & 26.9 \\
$\tau_{34}$ & 0.00005 & 0.00008 & 0.00005 & 0.00006 & 5.9 & 0.00031 & 0.00043 & 21.4 \\
$\tau_{44}$ & 0.00049 & 0.00084 & 0.00032 & 0.00057 & 8.2 & 0.00243 & 0.00512 & 33.0 \\
\hline & & & & & & & &
\end{tabular}

Model 1 and 2 in the same way as the simulation of Model 1 . The degrees of freedom $\delta$ in the prior distribution (2.14) was set to 7. However, when the Gibbs sampler did not work well, we used values of $\delta$ larger than 7 . For 10 samples constructed under $\delta=15$, we set $\delta=9$ in 3 out of 10 samples and $\delta=10$ in 1 sample. For the case $\delta=35$, we set $\delta=9$ in 1 sample; for the case $\delta=55$, we set $\delta=9$ in 1 sample.

Table 2(a), (b) and (c) collect results for the mean and standard deviation of the SE and the mean of the IF for each parameter. SEs and IFs for $\delta$ and $\boldsymbol{\Phi}$ are collected in Table 4.

The values of the IF obtained using Model 2, except for $\left\{\boldsymbol{\Sigma}_{c}\right\}$, are larger than the corresponding values obtained using Model 1 in Subsection 4.1. The sample of size 30,000 constructed by the Gibbs sampler corresponds to an independent sample of size not less than 300. The values of IF obtained using Model 3 are yet larger than the corresponding values obtained using Model 2. However, since we used the sample of size 200,000 to compute the posterior means, this result has little effect on convergence of the Markov chain.

For the regression-coefficient vector $\left\{\boldsymbol{\beta}_{c}\right\}$ and its hyperparameters $\boldsymbol{\mu}, \boldsymbol{T}$, which have no direct relationship with $\delta$, there are no significant differences in SE values between the three models. However, for large $\delta$, the SE values obtained 
Table 2(c). SEs and IFs of parameter estimates $(\delta=55)$.

\begin{tabular}{c|cc|ccc|ccr}
\hline & \multicolumn{2}{|c|}{ Model 1 } & \multicolumn{3}{c|}{ Model 2 } & \multicolumn{3}{c}{ Model 3 } \\
\hline & mean & s.d. & mean & s.d. & IF & mean & s.d. & \multicolumn{1}{c}{ IF } \\
\hline$\left\{\beta_{c 1}\right\}$ & 0.03291 & 0.00496 & 0.03474 & 0.00561 & 94.5 & 0.03352 & 0.00480 & 145.2 \\
$\left\{\beta_{c 2}\right\}$ & 0.03188 & 0.00269 & 0.03374 & 0.00371 & 91.1 & 0.03208 & 0.00261 & 144.4 \\
$\left\{\beta_{c 3}\right\}$ & 0.08569 & 0.00641 & 0.08907 & 0.00779 & 33.4 & 0.08683 & 0.00683 & 40.4 \\
$\left\{\beta_{c 4}\right\}$ & 0.08388 & 0.00912 & 0.08426 & 0.00906 & 34.8 & 0.08497 & 0.00967 & 47.3 \\
\hline$\left\{\sigma_{c 11}\right\}$ & 0.01884 & 0.00247 & 0.03182 & 0.00466 & 21.6 & 0.01949 & 0.00421 & 89.5 \\
$\left\{\sigma_{c 12}\right\}$ & 0.01689 & 0.00281 & 0.04051 & 0.00290 & 22.0 & 0.01726 & 0.00302 & 114.8 \\
$\left\{\sigma_{c 22}\right\}$ & 0.01884 & 0.00247 & 0.03182 & 0.00466 & 21.6 & 0.01949 & 0.00421 & 89.5 \\
\hline$\mu_{1}$ & 0.00096 & 0.00106 & 0.00107 & 0.00132 & 21.5 & 0.00124 & 0.00149 & 47.0 \\
$\mu_{2}$ & 0.00111 & 0.00106 & 0.00130 & 0.00125 & 20.2 & 0.00142 & 0.00164 & 43.9 \\
$\mu_{3}$ & 0.00143 & 0.00186 & 0.00250 & 0.00356 & 13.4 & 0.00204 & 0.00271 & 17.1 \\
$\mu_{4}$ & 0.00195 & 0.00344 & 0.00210 & 0.00365 & 13.1 & 0.00196 & 0.00408 & 19.7 \\
\hline$\tau_{11}$ & 0.00012 & 0.00012 & 0.00014 & 0.00021 & 28.2 & 0.00032 & 0.00036 & 53.5 \\
$\tau_{12}$ & 0.00012 & 0.00014 & 0.00011 & 0.00011 & 20.1 & 0.00028 & 0.00034 & 37.0 \\
$\tau_{13}$ & 0.00011 & 0.00013 & 0.00010 & 0.00008 & 19.3 & 0.00040 & 0.00060 & 26.5 \\
$\tau_{14}$ & 0.00014 & 0.00015 & 0.00012 & 0.00015 & 10.5 & 0.00042 & 0.00043 & 27.7 \\
$\tau_{22}$ & 0.00028 & 0.00020 & 0.00017 & 0.00016 & 22.8 & 0.00080 & 0.00063 & 54.0 \\
$\tau_{23}$ & 0.00014 & 0.00010 & 0.00013 & 0.00008 & 17.2 & 0.00057 & 0.00046 & 26.1 \\
$\tau_{24}$ & 0.00008 & 0.00007 & 0.00007 & 0.00006 & 10.1 & 0.00026 & 0.00025 & 28.4 \\
$\tau_{33}$ & 0.00037 & 0.00023 & 0.00025 & 0.00034 & 14.5 & 0.00192 & 0.00147 & 28.1 \\
$\tau_{34}$ & 0.00015 & 0.00011 & 0.00016 & 0.00014 & 6.2 & 0.00095 & 0.00088 & 22.7 \\
$\tau_{44}$ & 0.00038 & 0.00034 & 0.00026 & 0.00019 & 7.7 & 0.00219 & 0.00184 & 33.1 \\
\hline & & & & & & & & \\
\end{tabular}

Table 3. Mean values of the standard deviations of $\left\{\sigma_{c 11}\right\}$ and $\left\{\sigma_{c 12}\right\}$.

\begin{tabular}{l|ccc|ccc}
\hline & \multicolumn{3}{|c|}{$\sigma_{c 11}$} & \multicolumn{3}{c}{$\sigma_{c 12}$} \\
\hline & True value & Model 2 & Model 3 & True value & Model 2 & Model 3 \\
\hline$\delta=15$ & 0.239 & 0.148 & 0.089 & 0.234 & 0.144 & 0.085 \\
$\delta=35$ & 0.159 & 0.152 & 0.051 & 0.150 & 0.148 & 0.049 \\
$\delta=55$ & 0.126 & 0.148 & 0.048 & 0.121 & 0.147 & 0.046 \\
\hline
\end{tabular}

using Model 3 are slightly larger than the corresponding values obtained using Model 1, in which the covariance matrix is common to all individuals. Except for the case $\delta=55$, the mean value of the SE of $\left\{\boldsymbol{\Sigma}_{c}\right\}$ is smaller in Model 3 than in the other two models. In contrast, the estimation accuracy of Model 2 (which does not assume a hierarchical structure for the covariance matrix) is extremely poor.

Table 3 lists the standard deviations of the true and estimated values of $\left\{\sigma_{c 11}\right\}$ and $\left\{\sigma_{c 12}\right\}$, averaged over all samples.

As noted above, as $\delta$ increases, the standard deviations of $\left\{\sigma_{c 11}\right\}$ and $\left\{\sigma_{c 12}\right\}$ become small. Compared to these values, the standard deviations in the values estimated by Model 3 are extremely small. The phenomenon of estimates 
Table 4. Estimation results for $\delta$ and $\boldsymbol{\Phi}$.

\begin{tabular}{c|c|cc|r|cc}
\hline \multicolumn{2}{l|}{} & \multicolumn{2}{c|}{ SE } & \multicolumn{1}{c|}{ IF } & \multicolumn{2}{c}{ Estimates } \\
\hline \multicolumn{2}{l|}{} & mean & s.d. & mean & mean & s.d. \\
\hline$\delta=15$ & $\delta$ & 195.47 & 475.56 & 759.2 & 22.21 & 11.98 \\
& $\phi_{11}$ & 0.00348 & 0.00457 & 64.6 & 0.9739 & 0.0529 \\
& $\phi_{12}$ & 0.00181 & 0.00159 & 87.3 & 0.2962 & 0.0424 \\
\hline$\delta=35$ & $\delta$ & 319.80 & 351.92 & 671.0 & 43.78 & 15.58 \\
& $\phi_{11}$ & 0.00142 & 0.00203 & 77.9 & 1.0064 & 0.0371 \\
& $\phi_{12}$ & 0.00234 & 0.00208 & 95.9 & 0.2992 & 0.0483 \\
\hline$\delta=55$ & $\delta$ & 482.57 & 382.94 & 561.5 & 48.44 & 20.96 \\
& $\phi_{11}$ & 0.00303 & 0.00335 & 84.2 & 1.0190 & 0.0517 \\
& $\phi_{12}$ & 0.00185 & 0.00192 & 101.3 & 0.2952 & 0.0428 \\
\hline
\end{tabular}

approaching certain specific values is a characteristic feature of a shrinkage estimator, and is a result that could, to some extent, have been anticipated in view of our hypothesis of a hierarchical structure for $\boldsymbol{\Sigma}_{c}$. However, the extent of the shrinkage is larger than could have been anticipated. Returning to the discussion of Table 1 , as $\delta$ increases, the mean value of the SE in the values of $\left\{\boldsymbol{\Sigma}_{c}\right\}$ estimated by Model 3 becomes small and approaches the SE obtained in Model 1. A glance at Table 3 suggests that this observation does not so much indicate that the estimation accuracy is improving as it indicates that the estimates are approaching certain specific values.

Table 4 presents estimation results for $\delta$ and $\boldsymbol{\Phi}$.

We estimated the autocorrelations of $\delta$ and averaged these values over 10 samples. Figure 1 displays the averaged autocorrelation plot for $\delta$.

Figure 1 shows that the autocorrelation of $\delta$ decays to zero very slowly. The values of the IF of $\delta$ are extremely large. However, the sample to compute the posterior means corresponds to an independent sample of size not less than 260 .

Unfortunately, $\delta$ is estimated with extremely poor accuracy. This result could have been anticipated as a natural consequence of the fact that the standard deviation in the estimated values of $\left\{\boldsymbol{\Sigma}_{c}\right\}$ becomes excessively small as $\delta$ grows large. Indeed, as is clear from equations (2.7) through (2.9), in estimating $\delta$, our most important source of information is the variance of $\left\{\boldsymbol{\Sigma}_{c}\right\}$. We note that, in contrast to $\tilde{\boldsymbol{\Sigma}}_{c}$, both $V\left[\boldsymbol{\Sigma}_{c} \mid \delta, \boldsymbol{\Phi}\right]$ and $E\left[\boldsymbol{\Sigma}_{c} \mid \delta, \boldsymbol{\Phi}\right]$ depend on $\delta$.

Another factor that may be contributing somewhat to the poor accuracy with which $\delta$ is estimated is the fact that, in the prior distribution (2.15), we set the hyperparameter values to $\nu_{\delta}=1.5$ and $\psi_{\delta}=33$. However, even upon varying the values of the hyperparameters, we found little change in the estimation accuracy, as long as the hyperparameter values were chosen to ensure sufficiently large variance of $\delta$. The primary cause of the poor estimation accuracy is not the values of the hyperparameters or the small value of $C$, but rather the fact that the sample size per individual is set at the low value of $T_{c}=50$. Indeed, when we estimated $\delta$ and $\boldsymbol{\Phi}$ using $\left\{\boldsymbol{\Sigma}_{c}\right\}$ as our sample (by simply executing Steps 6 and 7 of the sampling algorithm), we found for the $C=200$ case that $\delta$ was estimated 


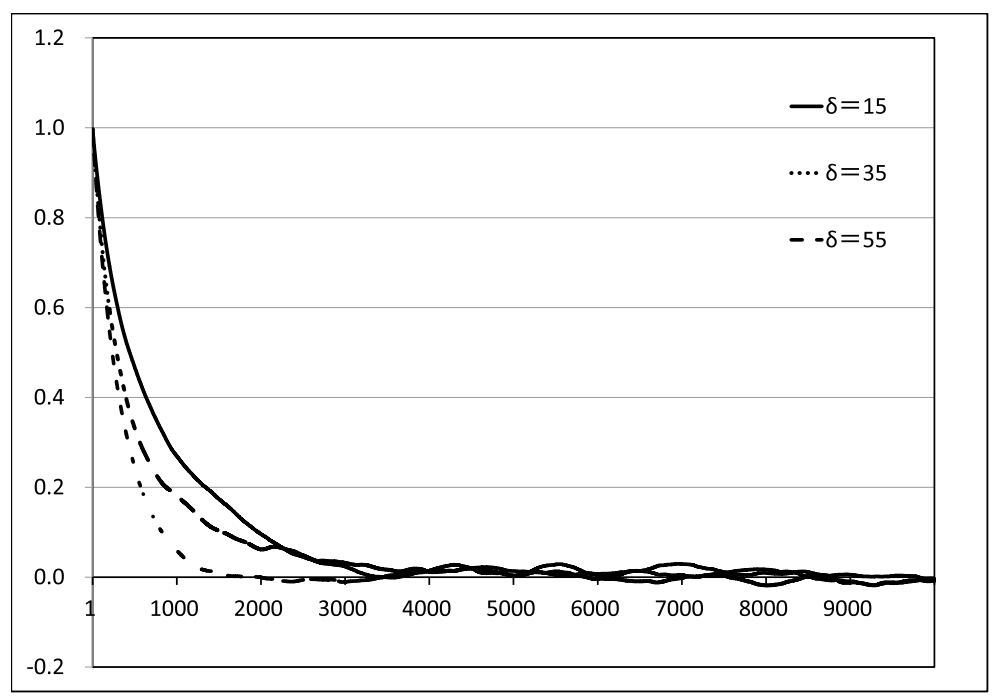

Figure 1. Autocorrelations of $\delta$.

to sufficiently high accuracy.

Although we were unable to run sufficient validation tests due to restrictions on computation time, we found (omitting the details) that the estimation accuracy in $\delta$ improved for $T_{c}=100$. However, the magnitude of the improvement was small, and the estimation accuracy remained insufficient. If we use a prior distribution that is close to being non-informative, then it seems unlikely that the estimation accuracy in $\delta$ could be improved without going to extremely large values of $T_{c}$ because, in the MNP model, only the results of choice can be observed.

\section{Empirical applications}

\subsection{Data}

In this section, we present the results of empirical applications using sales data for supermarkets located within the city of Tokyo. The data were gathered over a period of 408 days between April 27, 2009 and June 6, 2010, and the product categories which we examined were a yogurt and a special type of egg, which is deemed to be a value-added egg product, with the added value depending on factors such as the inclusion of large quantities of certain nutrients. For both product categories, the top three brands were considered in our analysis. The shares of three brands in our data are $6.2 \%, 5.2 \%$, and $4.9 \%$ for the case of the yogurt product, and $17.4 \%, 8.5 \%$, and $5.7 \%$ for the case of the egg product. The price is chosen as the covariate. Prices for each brand were normalized to have mean 0 and variance 1 .

Our analysis was restricted to customers who purchased items of two or more brands and who purchased a total of 20 or more items. For the yogurt product, the number of customers analyzed was 104, and the shares of the three brands 
among these customers were respectively $17.4 \%, 38.2 \%$, and $44.4 \%$. For the egg product, we had 77 customers, with shares of $65.6 \%, 6.3 \%$, and $28.1 \%$.

If, on a single shopping trip, a customer purchases 2 items from Brand 1 and 1 item from Brand 2, then we count 3 total cases of brand choice. Consequently, the sample size per customer is the total number of items purchased by that customer. For the yogurt product, the mean sample size was 45.3 ; the first quartile, median, third quartile, and maximum values were respectively 27,33 , 54, and 211. For the egg product, the corresponding numbers in order were $32.3,23,28,38$, and 87 . The final 5 items purchased by each customer were not used for estimation, but were used instead to test the accuracy of brand-choice predictions.

The hyperparameters in the prior distributions were assigned the same values as in the simulations. However, for Model 3, we set $d=9$ for the yogurt product and $d=8$ for the egg product, and for Model 2, we set $\delta=9$ and $\delta=8$, respectively. In Model 3, the range parameter $\epsilon_{\delta}$ of the uniform distribution from which $\delta$ is sampled was taken to have the value 4 for the yogurt product and 30 for the egg product.

\subsection{Estimation results}

Table 5(a) and (b) collects the means, standard deviations and inefficiency factors of $\left\{\boldsymbol{\beta}_{c}\right\}$ and $\left\{\boldsymbol{\Sigma}_{c}\right\}$ as estimated for each customer.

Table 6(a) and (b) detail the estimated values of $\boldsymbol{\mu}, \boldsymbol{T}, \delta$ and $\boldsymbol{\Phi}$.

Models 2 and 3 yield similar estimates for $\left\{\boldsymbol{\Sigma}_{c}\right\}$, whereas the differences between Models 2 and 3 in the estimated values of $\left\{\boldsymbol{\beta}_{c}\right\}$ and its hyperparameters $\boldsymbol{\mu}, \boldsymbol{T}$ are particularly large. For the case of the yogurt product, the estimated

Table 5(a). Estimation results for yogurt product.

\begin{tabular}{c|rcc|rcc}
\hline & \multicolumn{3}{|c|}{ Model 2 } & \multicolumn{3}{c}{ Model 3 } \\
\hline & mean & s.d. & IF & mean & s.d. & IF \\
\hline$\beta_{c 1}$ & -0.771 & 0.501 & 64.0 & -0.708 & 0.314 & 162.4 \\
$\beta_{2 c}$ & -1.229 & 1.394 & 66.4 & -1.779 & 2.381 & 236.1 \\
$\beta_{3 c}$ & -0.411 & 1.179 & 37.8 & -0.217 & 1.201 & 162.7 \\
\hline$\sigma_{11 c}$ & 1.044 & 0.151 & 13.6 & 1.504 & 0.091 & 177.6 \\
$\sigma_{12 c}$ & 0.429 & 0.095 & 11.5 & 0.460 & 0.058 & 186.2 \\
\hline
\end{tabular}

Table 5(b). Estimation results for egg product.

\begin{tabular}{c|rcc|rcc}
\hline & \multicolumn{3}{|c|}{ Model 2 } & \multicolumn{3}{c}{ Model 3 } \\
\hline & mean & s.d. & IF & mean & s.d. & IF \\
\hline$\beta_{c 1}$ & -0.547 & 0.423 & 48.1 & -0.549 & 0.302 & 144.6 \\
$\beta_{2 c}$ & 1.849 & 0.872 & 59.3 & 2.502 & 1.193 & 301.1 \\
$\beta_{3 c}$ & 0.672 & 1.086 & 72.0 & 1.189 & 2.170 & 283.3 \\
\hline$\sigma_{11 c}$ & 1.028 & 0.072 & 11.7 & 1.075 & 0.017 & 235.1 \\
$\sigma_{12 c}$ & 0.441 & 0.11 & 17.4 & 0.510 & 0.022 & 232.5 \\
\hline
\end{tabular}


Table 6(a). Estimation results for yogurt product.

\begin{tabular}{l|rr|rr|r|rc}
\hline & \multicolumn{2}{|c|}{ Model 2} & \multicolumn{5}{c}{ Model 3} \\
\hline & estimate & \multicolumn{1}{c|}{ IF } & estimate & \multicolumn{1}{c}{ IF } & & estimate & IF \\
\hline$\mu_{1}$ & -0.772 & 8.0 & -0.708 & 78.5 & $\delta$ & 9.355 & 158.9 \\
\cline { 5 - 7 }$\mu_{2}$ & -1.229 & 21.1 & -1.777 & 71.8 & $\phi_{11}$ & 1.524 & 163.4 \\
$\mu_{3}$ & -0.411 & 9.0 & -0.216 & 35.1 & $\phi_{12}$ & 0.484 & 174.7 \\
\hline$\tau_{11}$ & 0.380 & 38.0 & 0.176 & 89.1 & & & \\
$\tau_{12}$ & 0.148 & 11.3 & 0.239 & 119.3 & & & \\
$\tau_{13}$ & 0.097 & 18.7 & 0.192 & 96.3 & & & \\
$\tau_{22}$ & 2.232 & 10.8 & 6.741 & 134.7 & & & \\
$\tau_{23}$ & 0.403 & 13.2 & 1.741 & 113.6 & & & \\
$\tau_{33}$ & 1.589 & 8.3 & 1.676 & 120 & & & \\
\hline
\end{tabular}

Table 6(b). Estimation results for egg product.

\begin{tabular}{r|rr|rr|r|rr}
\hline & \multicolumn{2}{|c|}{ Model 2} & \multicolumn{5}{|c}{ Model 3} \\
\hline & estimate & \multicolumn{1}{c|}{ IF } & estimate & \multicolumn{1}{c}{ IF } & & estimate & IF \\
\hline$\mu_{1}$ & -0.548 & 9.2 & -0.549 & 87.1 & $\delta$ & 49.237 & 431.9 \\
\cline { 5 - 8 }$\mu_{2}$ & 1.850 & 23.7 & 2.501 & 195.6 & $\phi_{11}$ & 1.077 & 232.2 \\
$\mu_{3}$ & 0.671 & 32.4 & 1.187 & 133.5 & $\phi_{12}$ & 0.523 & 225.9 \\
\hline$\tau_{11}$ & 0.358 & 15.1 & 0.193 & 69.0 & & & \\
$\tau_{12}$ & -0.025 & 21.5 & 0.151 & 104.7 & & & \\
$\tau_{13}$ & 0.093 & 14.7 & 0.459 & 95.4 & & & \\
$\tau_{22}$ & 1.076 & 13.9 & 2.307 & 223.9 & & & \\
$\tau_{23}$ & 0.204 & 12.7 & 2.839 & 221.2 & & & \\
$\tau_{33}$ & 1.546 & 12.6 & 5.924 & 196.0 & & & \\
\hline
\end{tabular}

value of $\delta$ is small, and it indicates that the covariance matrix may differ considerably from individual to individual.

To investigate the goodness of the model fit, we compared the predictive accuracy of brand choice against validation data. The validation data were brandchoice results corresponding to the last 5 products purchased by each individual; as mentioned above, these data were not used for estimation purposes. The predictive accuracy for customer $c$ was assessed using the mean absolute error $\mathrm{MAE}_{c}$. The $\mathrm{MAE}_{c}$ is computed as the average, over 5 choice occasions, of the absolute error $\mathrm{AE}_{c t}$ of choice probability. The results of the $t$ th choice occasion by customer $c$ are expressed using indicator variables $u_{c t j}, j=1,2,3$. If Brand $j$ is purchased, then we set $u_{c t j}=1$ and $u_{c t k}=0, k \neq j$. The choice probability for the $t$ th choice occasion, as determined from the estimated values of $\boldsymbol{\beta}_{c}$ and $\boldsymbol{\Sigma}_{c}$, is denoted by $\hat{p}_{c t}(j), j=1,2,3$. Here, $\mathrm{AE}_{c t}$ is defined by

$$
\mathrm{AE}_{c t}=\frac{1}{3} \sum_{j=1}^{3}\left|u_{c t j}-\hat{p}_{c t}(j)\right|=\frac{2}{3}\left(1-\hat{p}_{c t}\left(Y_{c t}\right)\right) .
$$

The $\mathrm{MAE}_{c}$ averaged over all customers $c$ is denoted simply MAE. Table 7 collects 
Table 7. MAE values for each model.

\begin{tabular}{c|ccc}
\hline & Model 0 & Model 2 & Model 3 \\
\hline Yoghurt & 0.2627 & 0.2029 & 0.1939 \\
Egg & 0.2047 & 0.1679 & 0.1648 \\
\hline
\end{tabular}

the values of MAE obtained using the various models considered in this paper. The column labeled "Model 0" indicates the MAE computed by taking the brand choice probability to be simply the rate at which that brand was purchased during the estimation interval.

For both the yogurt and egg products, Model 3 exhibits the greatest predictive accuracy. We compute a difference between $\mathrm{MAE}_{c}$ by Model 2 and that by Model 3 for all customers. Taking these differences as a dataset and computing the $t$-test statistic against the null hypothesis that the population mean is 0 yields a value of -3.022 for the yogurt product, which is quite large in magnitude. The number of customers for whom the predictive accuracy increased (i.e., the number of customers for whom the difference in the mean absolute errors was negative) was 66, out of 104 total customers. Moreover, the regression coefficient for the price, $\beta_{c 1}$, should always evaluate to a negative number; the number of customers for which this quantity was estimated to be a positive number were 2 in Model 3, but 6 in Model 2. The improvement in predictive accuracy furnished by Model 3 is extremely small, but nonetheless is clearly statistically significant in the case of the yogurt product.

For the egg product, although some improvement in predictive accuracy is observed with Model 3, our results did not find this improvement to be statistically significant. One cause of this result may be the fact that the number of customers and the sample sizes were both smaller for the egg product than for the yogurt product. In the comparison of Model 3 to Model 2, the test statistic was -1.260 , and the number of customers for whom the predictive accuracy increased was 48 out of 77 . The number of customers for whom the estimated value of $\beta_{c 1}$ was positive were 3 for Model 3 and 8 for Model 2 .

Recently, an excellent new assessment criterion that is applicable to model selection in Bayes estimation, the widely applicable information criterion (WAIC), was proposed by Watanabe (2010). For the yogurt product, the values of WAIC were 3199.4 for Model 2 and 3108.3 for Model 3. For the egg product, the corresponding values were respectively 1657.3 and 1599.6.

Our numerical procedure for determining WAIC follows Gelman et al. (2013). They presented two methods for computing the functional variance; for the present study, we applied their recommended method that utilizes the variance of the log predictive density. In computing WAIC, we used the samples constructed by our Gibbs sampler. For both the yogurt and egg products, Model 3 exhibits the smallest WAIC. Although the difference in WAIC values between the two models is not large, Model 3 is clearly the optimal model. 


\section{Conclusions}

In this paper, we consider a complete HMNP model in which both the regression-coefficient vector and the covariance matrix are assumed to exhibit a hierarchical structure and propose an MCMC algorithm for numerically computing the Bayes estimates of the model parameters.

Simulations demonstrated that application of Model 3 proposed in this paper improves the accuracy in the estimation of the covariance matrix compared to Model 2 which does not assume a hierarchical structure for the covariance matrix. Comparisons with Model 1, in which the covariance matrix is common to all individuals, revealed the unsurprising conclusion that the accuracy of estimation is improved when the variance of the covariance matrices defined for each individual is large, whereas no improvement was observed when the variance was small. The reason for this is that we set $T_{c}$ to the small value of 50 . In applications of the HMNP model to actual data, one does not know whether the covariance matrices exhibit large variance, and thus, in practical problems, one should apply Model 3. In empirical applications, using Model 3 did lead to improvements in the predictive accuracy of brand choice.

In this study, $\delta$ is an important estimand. However, the estimation accuracy is extremely poor. As long as we use a prior distribution in Model 3 that is close to a non-informative distribution, this problem most likely cannot be overcome without going to extremely large values of $T_{c}$.

In our simulations, Model 3 was the correct model. However, as we have noted numerous times, when $T_{c}$ is small, there is no guarantee that the estimation accuracy obtained using Model 3 will exceed that obtained using the incorrect Model 1. In the present study, due to limited computation time, we were only able to run simulations with a restricted range of settings. However, practical applications of the HMNP model to real-world problems will require a more detailed investigation of these problems, one involving simulations conducted under a wider range of settings such as the sample sizes for each individual, the hyperparameters in the prior distribution, and the number of choice alternatives. These topics will be addressed in future work.

\section{Acknowledgements}

The author is grateful to two anonymous referees for helpful comments that improved this paper considerably.

\section{REFERENCES}

Allenby, G. M. and Rossi, P. E. (1999). Marketing models of consumer heterogeneity, J. Econom., 89, 57-78.

Anderson, T. W. (2003). An Introduction to Multivariate Statistical Analysis, 3rd ed., John Wiley.

Asano, F., Asoh, H. and Nakadai, K. (2013). Sound source localization using joint Bayesian estimation with a hierarchical noise model, IEEE Transactions on Audio, Speech, and Language Processing, 21, 1953-1965.

Bouriga, M. and Féron, O. (2013). Estimation of covariance matrices based on hierarchical inverse-Wishart priors, J. Stat. Plann. Inference, 143, 795-808. 
Burgette, L. F. and Nordheim, E. V. (2012). The trace restriction: An alternative identification strategy for the Bayesian multinomial probit model, J. Bus. Econ. Stat., 30, 404-410.

Gelman, A., Hwangz, J. and Vehtari, A. (2013). Understanding predictive information criteria for Bayesian models, Stat. Comput., published online: 20 August 2013.

Geweke, J. (1992). Evaluating the accuracy of sampling-based approaches to the calculation of posterior moments, Bayesian Statistics (eds. J. M. Bernardo, J. O. Berger, A. P. Dawid and A. F. M. Smith), 4, 169-193, Oxford University Press, Oxford.

Haff, L. R. (1979). An identity for the Wishart distribution with applications, J. Multivar. Anal., 9, 531-544.

Imai, K. and van Dyk, D. A. (2005). A Bayesian analysis of the multinomial probit model using marginal data augmentation, J. Econom., 124, 311-334.

Matsumae, T., Iiboshi, H., Namba, R. and Nishiyama, S. (2011). An application of hybrid MCMC and simulation smoother in estimating a DSGE Model with measurement errors, J. Japan Statist. Soc. Japanese Issue, 41(1), 83-121.

McCulloch, R. and Rossi, P. E. (1994). An exact likelihood analysis of the multinomial probit model, J. Econom., 64, 217-228.

McCulloch, R., Polson, N. and Rossi, P. E. (2000). Bayesian analysis of the multinomial probit model with fully identified parameters, J. Econom., 99, 173-193.

Meng, X.-L. and van Dyk, D. A. (1999). Seeking efficient data augmentation schemes via conditional and marginal augmentation, Biometrika, 86, 301-320.

Nobile, A. (1998). A hybrid Markov chain for the Bayesian analysis of the multinomial probit model, Stat. Comput., 8, 229-242.

Rossi, P. E., McCulloch, R. E. and Allenby, G. M. (1996). The value of purchase history data in target marketing, Mark. Sci., 15, 321-340.

Watanabe, S. (2010). Asymptotic equivalence of Bayes cross validation and widely applicable information criterion in singular learning theory, J. Mach. Learn. Res., 11, 3571-3549. 